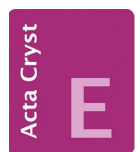

CRYSTALLOGRAPHIC COMMUNICATIONS

ISSN 2056-9890

Received 2 March 2016

Accepted 16 March 2016

Edited by R. F. Baggio, Comisión Nacional de Energía Atómica, Argentina

Keywords: crystal structure; imidazole derivative; hydrogen bonding; supramolecular architecture.

CCDC reference: 1468833

Supporting information: this article has supporting information at journals.iucr.org/e

\section{Crystal structure of 1,2-bis[(1H-imidazol-2-yl)- methylidene]hydrazine and its one-dimensional hydrogen-bonding network}

\author{
Chia-Hwa Lee $^{\mathrm{a}}$ and Gene-Hsiang Lee ${ }^{\mathrm{b} *}$
}

${ }^{\mathbf{a}}$ Department of Chemistry, National Taiwan University, Taipei, Taiwan, and ${ }^{\mathbf{b}}$ Instrumentation Center, National Taiwan University, Taipei, Taiwan. *Correspondence e-mail: ghlee@ntu.edu.tw

In the title compound, $\mathrm{C}_{8} \mathrm{H}_{8} \mathrm{~N}_{6}$, two imidazolyl groups are separated by a zigzag $-\mathrm{CH}=\mathrm{N}-\mathrm{N}=\mathrm{CH}-$ linkage. An inversion center is located at the mid-point of the $\mathrm{N}-\mathrm{N}$ single bond and the complete molecule is generated by symmetry. In the crystal, each molecule forms four $\mathrm{N}-\mathrm{H} \cdots \mathrm{N}$ hydrogen bonds with two neighbouring molecules to constitute a one-dimensional ladder-like structure propagating along the $a$-axis direction.

\section{Chemical context}

Supramolecular chemistry is a fascinating topic, and molecular assemblies via intermolecular non-covalent binding interactions (i.e. hydrogen bonding, ionic and $\pi-\pi$ stacking interactions) have attracted much attentions in the field of crystal engineering over the last decade. In particular, hydrogen bonding, which is a powerful organizing force in designing a variety of supramolecular and solid-state architectures (Subramanian \& Zaworotko, 1994), is not only used extensively to generate numerous network structures consisting of discrete organic and organometallic compounds (Desiraju, 2000), but is also responsible for interesting physical properties of these supramolecular arrangements, such as electrical, optical, magnetic, etc. (Bacchi \& Pelagatti, 2016; Lindoy \& Atkinson, 2000; Létard et al., 1998).

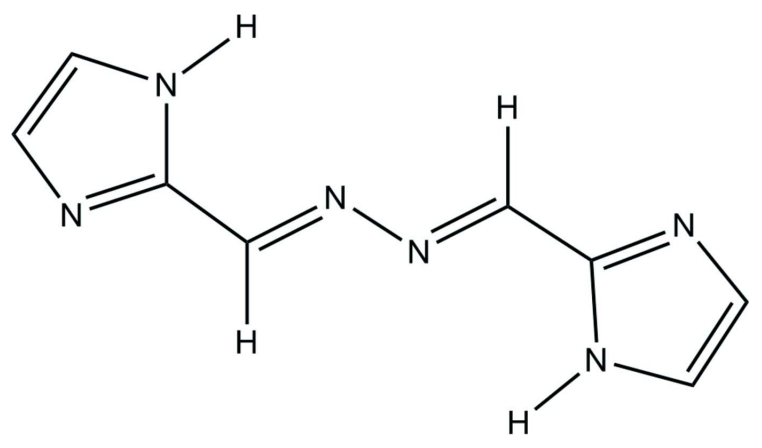

Imidazoles, containing two nitrogen atoms, possess both hydrogen-bond donating and accepting sites and are superior building blocks for supramolecular architectures. Many imidazole-containing polydentate ligands derived from hydrazine find a wide range of applications in coordination chemistry owing to their chelating ability (Zhou et al., 2012). In this paper we report the synthesis of 1,2-bis[( $1 H$-imidazol2-yl)methylene]hydrazine (I), designed to consist of nitrogen donors and acceptors, and the supramolecular architecture it 


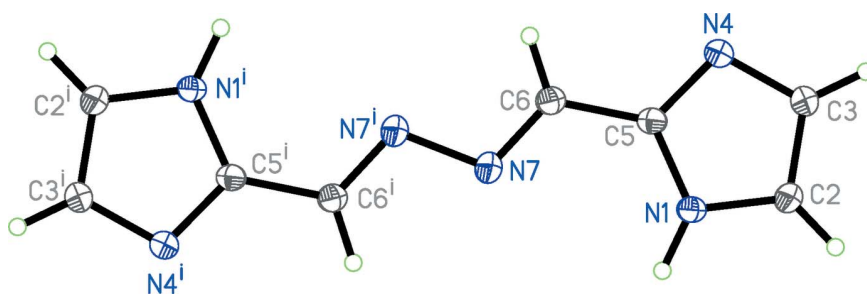

Figure 1

The molecular structure of (I), showing the atom-labeling scheme. Displacement ellipsoids are drawn at the $50 \%$ probability level. [Symmetry code: (i) $-x,-y+1,-z+2$.]

gives rise to via hydrogen bonds. The functionality of molecule (I) as a bridge between metal centers for the formation of multi-dimensional structures will be discussed in subsequent publications.

\section{Structural commentary}

The molecular structure of the title compound consists of two imidazolyl groups linked by a zigzag $-\mathrm{CH}=\mathrm{N}-\mathrm{N}=\mathrm{CH}-$ linkage (Fig. 1) and with C5 $\cdots C 5^{\mathrm{i}}=5.937$ (3) $\AA$ [the distance between the centroids of the imidazolyl groups is 8.103 (3) $\AA$ ]. The molecule possesses an inversion center located in the midpoint of the $\mathrm{N}-\mathrm{N}$ single bond and the complete molecule is generated by symmetry. The molecule appears in a $Z(E E) Z$ configuration and its geometry is similar to that of 1,2-bis[ $(1 \mathrm{H}$ imidazol-5-yl)methylene]hydrazine (Pinto et al., 2013) and 1,2bis[(thiophene-3-yl)methylene]hydrazine (Kim \& Lee, 2008).

The molecule $(\mathrm{I})$ has a planar (r.m.s. deviation $=0.012 \AA$ ) structure which, in addition to the observed bond distances, suggests partial delocalization of the $\pi$ electrons over the

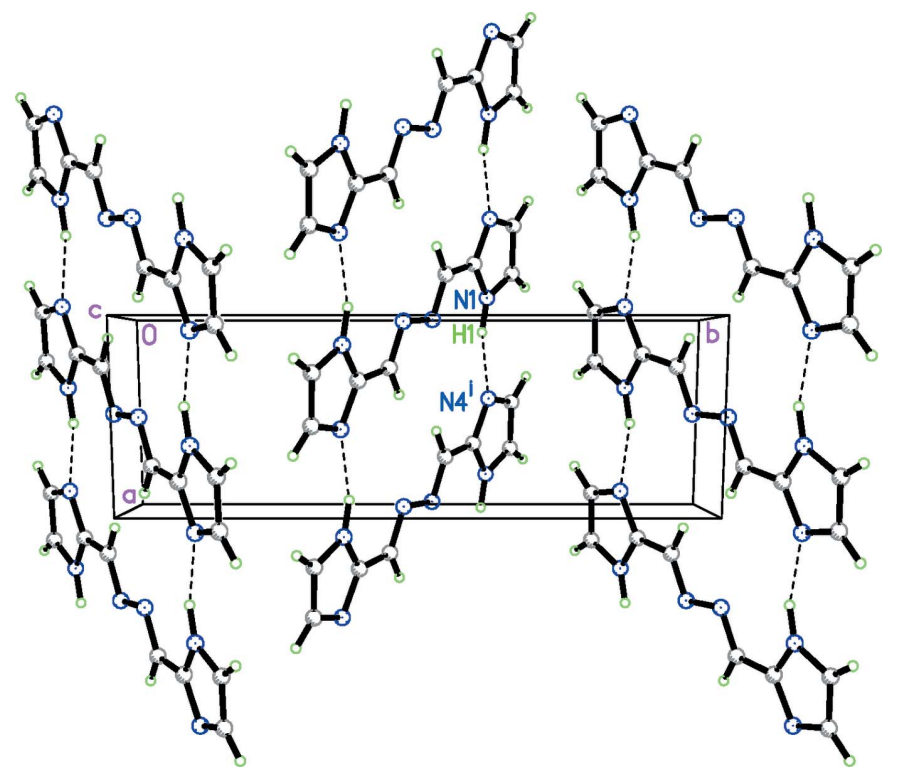

Figure 2

A packing diagram for (I), viewed along the $c$ axis. Dashed lines represent hydrogen bonds. [Symmetry code: (i) $x+1, y, z$.]
Table 1

Hydrogen-bond geometry $\left(\AA{ }^{\circ}{ }^{\circ}\right)$.

\begin{tabular}{lllll}
\hline$D-\mathrm{H} \cdots A$ & $D-\mathrm{H}$ & $\mathrm{H} \cdots A$ & $D \cdots A$ & $D-\mathrm{H} \cdots A$ \\
\hline $\mathrm{N} 1-\mathrm{H} 1 \cdots \mathrm{N} 4^{\mathrm{i}}$ & $0.95(2)$ & $1.95(2)$ & $2.8493(17)$ & $157.9(19)$ \\
\hline
\end{tabular}

Symmetry code: (i) $x+1, y, z$.

whole molecule. The geometric parameters, viz., the $\mathrm{N}-\mathrm{N}$ single bond $\left[\mathrm{N} 7-\mathrm{N} 7^{\mathrm{i}}=1.409\right.$ (2) $\AA$; symmetry code: $(\mathrm{i})-x$, $-y+1,-z+2], \mathrm{C}=\mathrm{N}$ double bond [C6-N7 = 1.2795 (19) $\AA$ ] and $\mathrm{C}=\mathrm{N}-\mathrm{N}$ bond angle $\left[\mathrm{C} 6=\mathrm{N} 7-\mathrm{N} 7^{\mathrm{i}}=111.41(15)^{\circ}\right]$, are comparable to the corresponding parameters found in 1,4bis(3-pyridyl)-2,3-diaza-1,3-butadiene [Dong et al., 2000] and 1,4-bis(4-pyridyl)-2,3-diaza-1,3-butadiene [Ciurtin et al., 2001].

\section{Supramolecular features}

In the crystal structure of (I), each molecule is involved in four $\mathrm{N}-\mathrm{H} \cdots \mathrm{N}$ hydrogen bonds (i.e.: two donor and two acceptor interactions) and interacts with two neighboring molecules, resulting in a one-dimensional ladder-like structure along the $a$ axis (Fig. 2). Numerical details of the hydrogen-bonding geometry are tabulated in Table 1 .

As a comparison, the related compound 1,2-bis[(1Himidazol-5-yl)methylene]hydrazine (Pinto et al., 2013) is a planar molecule which constitutes corrugated layers parallel

Table 2

Experimental details.

\begin{tabular}{|c|c|}
\hline Crystal data & \\
\hline Chemical formula & $\mathrm{C}_{8} \mathrm{H}_{8} \mathrm{~N}_{6}$ \\
\hline$M_{\mathrm{r}}$ & 188.20 \\
\hline Crystal system, space group & Monoclinic, $P 2_{1} / n$ \\
\hline Temperature $(\mathrm{K})$ & 150 \\
\hline$a, b, c(\AA)$ & $5.0618(3), 14.6282(8), 6.1294(4)$ \\
\hline$\beta\left({ }^{\circ}\right)$ & $106.321(2)$ \\
\hline$V\left(\AA^{3}\right)$ & $435.56(5)$ \\
\hline$Z$ & 2 \\
\hline Radiation type & Мо $K \alpha$ \\
\hline$\mu\left(\mathrm{mm}^{-1}\right)$ & 0.10 \\
\hline Crystal size $(\mathrm{mm})$ & $0.35 \times 0.10 \times 0.03$ \\
\hline Data collection & \\
\hline Diffractometer & Bruker D8 VENTURE \\
\hline Absorption correction & $\begin{array}{l}\text { Multi-scan (SADABS; Bruker, } \\
\text { 2015) }\end{array}$ \\
\hline$T_{\min }, T_{\max }$ & $0.702,0.746$ \\
\hline $\begin{array}{l}\text { No. of measured, independent and } \\
\text { observed }[I>2 \sigma(I)] \text { reflections }\end{array}$ & $2614,999,903$ \\
\hline$R_{\text {int }}$ & 0.014 \\
\hline$(\sin \theta / \lambda)_{\max }\left(\AA^{-1}\right)$ & 0.650 \\
\hline Refinement & \\
\hline$R\left[F^{2}>2 \sigma\left(F^{2}\right)\right], w R\left(F^{2}\right), S$ & $0.042,0.117,1.12$ \\
\hline No. of reflections & 999 \\
\hline No. of parameters & 68 \\
\hline $\mathrm{H}$-atom treatment & $\begin{array}{l}\mathrm{H} \text { atoms treated by a mixture of } \\
\text { independent and constrained } \\
\text { refinement }\end{array}$ \\
\hline$\Delta \rho_{\max }, \Delta \rho_{\min }\left(\mathrm{e} \AA^{-3}\right)$ & $0.31,-0.26$ \\
\hline
\end{tabular}

Computer programs: APEX3 and SAINT (Bruker, 2015), SHELXS97 and SHELXTL (Sheldrick, 2008) and SHELXL2014 (Sheldrick, 2015). 
to the (101) plane, as a result of both hydrogen bonding and $\pi-\pi$ stacking interactions with adjacent molecules. In the present case of (I), instead, there are no significant $\pi-\pi$ stacking interactions.

\section{Synthesis and crystallization}

A methanol solution $(10 \mathrm{~mL})$ of imidazole-2-carboxaldehyde $(2.48 \mathrm{~g}, 25.8 \mathrm{mmol})$ was added to a methanol solution $(10 \mathrm{~mL})$ of hydrazine monohydrate $(0.64 \mathrm{ml}, 12.9 \mathrm{mmol})$. The mixture was stirred for $3 \mathrm{~h}$ and the precipitate was collected by filtration. Single crystals suitable for X-ray diffraction studies were obtained by diffusion of diethyl ether into a DMSO solution of the title compound (I). Yield: $2.21 \mathrm{~g}$ (91\%).

\section{Refinement}

Crystal data, data collection and structure refinement details are summarized in Table 2. All the $\mathrm{H}$ atoms were located in difference-Fourier maps. For the $\mathrm{H}$ atom bounded to atom $\mathrm{N} 1$, the atomic coordinates and $U_{\text {iso }}$ were refined, giving an $\mathrm{N}-\mathrm{H}$ distance of 0.95 (2) $\AA$. The $\mathrm{C}$-bound $\mathrm{H}$ atoms were subsequently treated as riding atoms in geometrically idealized positions: $\mathrm{C}-\mathrm{H}$ distances of $0.95 \AA$ with $U_{\text {iso }}(\mathrm{H})=$ $1.2 U_{\text {eq }}(\mathrm{C})$.

\section{Acknowledgements}

GHL thanks the Instrumentation Center, National Taiwan University, for support of this work.

\section{References}

Bacchi, A. \& Pelagatti, P. (2016). Chem. Commun. 52, 1327-1337.

Bruker (2015). APEX3, SAINT and SADABS. Bruker AXS Inc, Madison, Wisconsin, USA.

Ciurtin, D. M., Dong, Y.-B., Smith, M. D., Barclay, T. \& zur Loye, H.-C. (2001). Inorg. Chem. 40, 2825-2834.

Desiraju, G. R. (2000). Stimulating Concepts in Chemistry, edited by F. Vogtle, J. F. Stoddart \& M. Shibasaki, pp. 293-302. Weinheim: Wiley VCH.

Dong, Y.-B., Smith, M. D., Layland, R. C. \& zur Loye, H.-C. (2000). Chem. Mater. 12, 1156-1161.

Kim, S. H. \& Lee, S. W. (2008). Inorg. Chim. Acta, 361, 137-144.

Létard, J. F., Guionneau, P., Rabardel, L., Howard, J. A. K., Goeta, A. E., Chasseau, D. \& Kahn, O. (1998). Inorg. Chem. 37, 4432-4441.

Lindoy, L. F. \& Atkinson, I. M. (2000). In Self-assembly in Supramolecular Systems, pp. 8-46. Cambridge: RSC.

Pinto, J., Silva, V. L. M., Silva, A. M. S., Claramunt, R. M., Sanz, D., Torralba, M. C., Torres, M. R., Reviriego, F., Alkorta, I. \& Elguero, J. (2013). Magn. Reson. Chem. 51, 203-221.

Sheldrick, G. M. (2008). Acta Cryst. A64, 112-122.

Sheldrick, G. M. (2015). Acta Cryst. C71, 3-8.

Subramanian, S. \& Zaworotko, M. J. (1994). Coord. Chem. Rev. 137, 357-401.

Zhou, X.-P., Li, M., Liu, J. \& Li, D. (2012). J. Am. Chem. Soc. 134, 6770. 


\section{supporting information}

Acta Cryst. (2016). E72, 556-558 [https://doi.org/10.1107/S2056989016004497]

\section{Crystal structure of 1,2-bis[(1H-imidazol-2-yl)methylidene]hydrazine and its one-dimensional hydrogen-bonding network}

\section{Chia-Hwa Lee and Gene-Hsiang Lee}

Computing details

Data collection: APEX3 (Bruker, 2015); cell refinement: SAINT (Bruker, 2015); data reduction: SAINT (Bruker, 2015); program(s) used to solve structure: SHELXS97 (Sheldrick, 2008); program(s) used to refine structure: SHELXL2014 (Sheldrick, 2015); molecular graphics: SHELXTL (Sheldrick, 2008); software used to prepare material for publication: SHELXTL (Sheldrick, 2008).

1,2-Bis[(1H-imidazol-2-yl)methylidene $]$ hydrazine

Crystal data

$\mathrm{C}_{8} \mathrm{H}_{8} \mathrm{~N}_{6}$

$M_{r}=188.20$

Monoclinic, $P 2_{1} / n$

$a=5.0618(3) \AA$

$b=14.6282(8) \AA$

$c=6.1294(4) \AA$

$\beta=106.321(2)^{\circ}$

$V=435.56(5) \AA^{3}$

$Z=2$

$F(000)=196$

$D_{\mathrm{x}}=1.435 \mathrm{Mg} \mathrm{m}^{-3}$

Mo $K \alpha$ radiation, $\lambda=0.71073 \AA$

Cell parameters from 2074 reflections

$\theta=2.8-27.5^{\circ}$

$\mu=0.10 \mathrm{~mm}^{-1}$

$T=150 \mathrm{~K}$

Needle, colourless

$0.35 \times 0.10 \times 0.03 \mathrm{~mm}$

\section{Data collection}

Bruker D8 VENTURE diffractometer

$\varphi$ and $\omega$ scans

Absorption correction: multi-scan

(SADABS; Bruker, 2015)

$T_{\min }=0.702, T_{\max }=0.746$

2614 measured reflections

999 independent reflections

903 reflections with $I>2 \sigma(I)$

$R_{\text {int }}=0.014$

$\theta_{\text {max }}=27.5^{\circ}, \theta_{\min }=2.8^{\circ}$

$h=-6 \rightarrow 6$

$k=-18 \rightarrow 19$

$l=-7 \rightarrow 6$

\section{Refinement}

Refinement on $F^{2}$

Least-squares matrix: full

$R\left[F^{2}>2 \sigma\left(F^{2}\right)\right]=0.042$

$w R\left(F^{2}\right)=0.117$

$S=1.12$

999 reflections

68 parameters

0 restraints

Hydrogen site location: mixed

$\mathrm{H}$ atoms treated by a mixture of independent and constrained refinement

$w=1 /\left[\sigma^{2}\left(F_{\mathrm{o}}^{2}\right)+(0.0528 P)^{2}+0.2863 P\right]$ where $P=\left(F_{\mathrm{o}}^{2}+2 F_{\mathrm{c}}^{2}\right) / 3$

$(\Delta / \sigma)_{\max }<0.001$

$\Delta \rho_{\max }=0.31 \mathrm{e} \AA^{-3}$

$\Delta \rho_{\min }=-0.26$ e $\AA^{-3}$ 


\section{Special details}

Geometry. All esds (except the esd in the dihedral angle between two 1.s. planes) are estimated using the full covariance matrix. The cell esds are taken into account individually in the estimation of esds in distances, angles and torsion angles; correlations between esds in cell parameters are only used when they are defined by crystal symmetry. An approximate (isotropic) treatment of cell esds is used for estimating esds involving l.s. planes.

Fractional atomic coordinates and isotropic or equivalent isotropic displacement parameters $\left(\AA^{2}\right)$

\begin{tabular}{lllll}
\hline & $x$ & $y$ & $z$ & $U_{\text {iso }} * / U_{\text {eq }}$ \\
\hline N1 & $-0.1374(2)$ & $0.62851(8)$ & $0.4802(2)$ & $0.0175(3)$ \\
H1 & $0.055(5)$ & $0.6193(14)$ & $0.515(4)$ & $0.037(6)^{*}$ \\
C2 & $-0.2801(3)$ & $0.67725(10)$ & $0.2944(2)$ & $0.0204(4)$ \\
H2 & -0.2082 & 0.7044 & 0.1823 & $0.025^{*}$ \\
C3 & $-0.5481(3)$ & $0.67920(10)$ & $0.3022(2)$ & $0.0197(3)$ \\
H3 & -0.6956 & 0.7084 & 0.1937 & $0.024^{*}$ \\
N4 & $-0.5714(2)$ & $0.63279(8)$ & $0.4899(2)$ & $0.0187(3)$ \\
C5 & $-0.3193(3)$ & $0.60313(10)$ & $0.5944(2)$ & $0.0162(3)$ \\
C6 & $-0.2539(3)$ & $0.55035(10)$ & $0.8020(2)$ & $0.0180(3)$ \\
H6 & -0.3975 & 0.5336 & 0.8659 & $0.022^{*}$ \\
N7 & $-0.0068(3)$ & $0.52574(8)$ & $0.9014(2)$ & $0.0195(3)$ \\
\hline
\end{tabular}

Atomic displacement parameters $\left(\AA^{2}\right)$

\begin{tabular}{lllllll}
\hline & $U^{11}$ & $U^{22}$ & $U^{33}$ & $U^{12}$ & $U^{13}$ & $U^{23}$ \\
\hline $\mathrm{N} 1$ & $0.0139(6)$ & $0.0223(6)$ & $0.0170(6)$ & $0.0001(5)$ & $0.0052(5)$ & $0.0008(5)$ \\
$\mathrm{C} 2$ & $0.0187(7)$ & $0.0264(8)$ & $0.0165(7)$ & $0.0001(6)$ & $0.0055(5)$ & $0.0039(5)$ \\
$\mathrm{C} 3$ & $0.0168(7)$ & $0.0227(7)$ & $0.0187(7)$ & $0.0013(5)$ & $0.0037(5)$ & $0.0040(5)$ \\
$\mathrm{N} 4$ & $0.0152(6)$ & $0.0220(6)$ & $0.0188(6)$ & $0.0006(5)$ & $0.0046(5)$ & $0.0027(5)$ \\
$\mathrm{C} 5$ & $0.0141(7)$ & $0.0180(7)$ & $0.0167(7)$ & $-0.0007(5)$ & $0.0049(5)$ & $-0.0013(5)$ \\
C6 & $0.0166(7)$ & $0.0205(7)$ & $0.0173(7)$ & $-0.0005(5)$ & $0.0054(5)$ & $0.0004(5)$ \\
N7 & $0.0198(6)$ & $0.0224(6)$ & $0.0159(6)$ & $0.0003(5)$ & $0.0043(5)$ & $0.0030(5)$ \\
\hline
\end{tabular}

Geometric parameters $\left(\AA,{ }^{\circ}\right)$

\begin{tabular}{llll}
\hline $\mathrm{N} 1-\mathrm{C} 5$ & $1.3557(18)$ & $\mathrm{C} 3-\mathrm{H} 3$ & 0.9500 \\
$\mathrm{~N} 1-\mathrm{C} 2$ & $1.3656(18)$ & $\mathrm{N} 4-\mathrm{C} 5$ & $1.3295(18)$ \\
$\mathrm{N} 1-\mathrm{H} 1$ & $0.95(2)$ & $\mathrm{C} 5-\mathrm{C} 6$ & $1.445(2)$ \\
$\mathrm{C} 2-\mathrm{C} 3$ & $1.371(2)$ & $\mathrm{C} 6-\mathrm{N} 7$ & $1.2795(19)$ \\
$\mathrm{C} 2-\mathrm{H} 2$ & 0.9500 & $\mathrm{C} 6-\mathrm{H} 6$ & 0.9500 \\
$\mathrm{C} 3-\mathrm{N} 4$ & $1.3689(19)$ & $\mathrm{N} 7-\mathrm{N} 7^{\mathrm{i}}$ & $1.409(2)$ \\
& & & \\
$\mathrm{C} 5-\mathrm{N} 1-\mathrm{C} 2$ & $107.26(12)$ & $\mathrm{C} 5-\mathrm{N} 4-\mathrm{C} 3$ & $105.60(12)$ \\
$\mathrm{C} 5-\mathrm{N} 1-\mathrm{H} 1$ & $130.4(13)$ & $\mathrm{N} 4-\mathrm{C} 5-\mathrm{N} 1$ & $111.18(13)$ \\
$\mathrm{C} 2-\mathrm{N} 1-\mathrm{H} 1$ & $122.3(13)$ & $\mathrm{N} 4-\mathrm{C} 5-\mathrm{C} 6$ & $123.37(13)$ \\
$\mathrm{N} 1-\mathrm{C} 2-\mathrm{C} 3$ & $106.15(12)$ & $\mathrm{N} 1-\mathrm{C} 5-\mathrm{C} 6$ & $125.46(13)$ \\
$\mathrm{N} 1-\mathrm{C} 2-\mathrm{H} 2$ & 126.9 & $\mathrm{~N} 7-\mathrm{C} 6-\mathrm{C} 5$ & 119.3 \\
$\mathrm{C} 3-\mathrm{C} 2-\mathrm{H} 2$ & 126.9 & $\mathrm{~N} 7-\mathrm{C} 6-\mathrm{H} 6$ & 119.3 \\
$\mathrm{~N} 4-\mathrm{C} 3-\mathrm{C} 2$ & $109.81(13)$ & $\mathrm{C} 5-\mathrm{C} 6-\mathrm{H} 6$ &
\end{tabular}




$\begin{array}{llll}\mathrm{N} 4-\mathrm{C} 3-\mathrm{H} 3 & 125.1 & \mathrm{C} 6-\mathrm{N} 7-\mathrm{N} 7^{\mathrm{i}} & 111.41(15) \\ \mathrm{C} 2-\mathrm{C} 3-\mathrm{H} 3 & 125.1 & & \\ \mathrm{C} 5-\mathrm{N} 1-\mathrm{C} 2-\mathrm{C} 3 & -0.35(16) & \mathrm{C} 2-\mathrm{N} 1-\mathrm{C} 5-\mathrm{N} 4 & 0.38(17) \\ \mathrm{N} 1-\mathrm{C} 2-\mathrm{C} 3-\mathrm{N} 4 & 0.21(17) & \mathrm{C} 2-\mathrm{N} 1-\mathrm{C} 5-\mathrm{C} 6 & -179.95(14) \\ \mathrm{C} 2-\mathrm{C} 3-\mathrm{N} 4-\mathrm{C} 5 & 0.02(17) & \mathrm{N} 4-\mathrm{C} 5-\mathrm{C} 6-\mathrm{N} 7 & -177.58(13) \\ \mathrm{C} 3-\mathrm{N} 4-\mathrm{C} 5-\mathrm{N} 1 & -0.25(16) & \mathrm{N} 1-\mathrm{C} 5-\mathrm{C} 6-\mathrm{N} 7 & 2.8(2) \\ \mathrm{C} 3-\mathrm{N} 4-\mathrm{C} 5-\mathrm{C} 6 & -179.93(13) & \mathrm{C} 5-\mathrm{C} 6-\mathrm{N} 7-\mathrm{N} 7^{\mathrm{i}} & -179.35(14)\end{array}$

Symmetry code: (i) $-x,-y+1,-z+2$.

Hydrogen-bond geometry $\left(\AA,{ }^{\circ}\right)$

\begin{tabular}{lllll}
\hline$D-\mathrm{H} \cdots A$ & $D-\mathrm{H}$ & $\mathrm{H} \cdots A$ & $D \cdots A$ & $D-\mathrm{H} \cdots A$ \\
\hline $\mathrm{N} 1-\mathrm{H} 1 \cdots \mathrm{N} 4^{\mathrm{ii}}$ & $0.95(2)$ & $1.95(2)$ & $2.8493(17)$ & $157.9(19)$
\end{tabular}

Symmetry code: (ii) $x+1, y, z$. 